\title{
Probing the Galactic Bulge with deep Adaptive Optics imaging: the age of NGC $6440^{1}$
}

\author{
L. Origlia ${ }^{2}$, S. Lena ${ }^{3}$, E. Diolaiti², F.R. Ferraro ${ }^{3}$,E. Valenti ${ }^{4}$, S. Fabbri ${ }^{3}$, G. Beccari ${ }^{3}$
}

\begin{abstract}
We present first results of a pilot project aimed at exploiting the potentiality of ground based adaptive optics imaging in the near infrared to determine the age of stellar clusters in the Galactic Bulge. We have used a combination of high resolution adaptive optics (ESO-VLT NAOS-CONICA) and wide-field (ESO-NTT-SOFI) photometry of the metal rich globular cluster NGC 6440 located towards the inner Bulge, to compute a deep color magnitude diagram from the tip of the Red Giant Branch down to $J \sim 22$, two magnitudes below the Main Sequence Turn Off (TO). The magnitude difference between the TO level and the red Horizontal Branch has been used as an age indicator. It is the first time that such a measurement for a bulge globular cluster has been obtained with a ground based telescope. From a direct comparison with 47 Tuc and with a set of theoretical isochrones, we concluded that NGC 6440 is old and likely coeval to 47 Tuc. This result adds a new evidence that the Galactic Bulge is $\approx 2$ Gyr younger at most than the pristine, metal poor population of the Galactic Halo.
\end{abstract}

Subject headings: Globular clusters: individual (NGC 6440); stars: evolution -

\section{Introduction}

Galactic globular clusters (GCs) are known since a long time to be the oldest stellar population of our Galaxy, hence their accurate age determination is crucial to settle the

\footnotetext{
${ }^{1}$ Based on observations collected at the European Southern Observatory (programs 075.D-0359(A) and 077.D-0316(A)), at the VLT, Paranal and program 081.D-0371(A) at NTT, La Silla, Chile.

${ }^{2}$ INAF - Osservatorio Astronomico di Bologna, via Ranzani 1, I-40127 Bologna, Italy, livia.origlia oabo.inaf.it, emiliano.diolaiti oabo.inaf.it, giacomo.beccari oabo.inaf.it

${ }^{3}$ Dipartimento di Astronomia, Università degli Studi di Bologna, via Ranzani 1, I-40127 Bologna, Italy, sebastiano.lena studio.unibo.it, francesco.ferraro3 unibo.it, sara.fabbri5 studio.unibo.it

${ }^{4}$ European Southern Observatory, Alonso de Cordova 3107, Vitacura, Santiago, Chile, evalenti eso.org
} 
epoch of the Galaxy formation and a lower limit to the age of the Universe. A major effort has been devoted to obtain reliable ages for the Halo GC system (Rosenberg et al. 1999; Rosenberg 2000; Salaris \& Weiss 2002; De Angeli et al. 2005).

With the growing awareness that the Bulge GCs are a distinct sub-system, has come a new urgency to properly characterize their evolutionary sequences and to determine their ages and chemical composition. Moreover, Bulge GCs are key templates of simple stellar populations to study the stellar and chemical evolution in the high metallicity domain (McWilliam 1997; Matteucci et al. 1999; Wyse et al. 2000; Ballero et al. 2007) and for population synthesis of giant elliptical galaxies. Hence, a proper characterization of the main chemical and evolutionary properties of the Galactic Bulge and its comparison with the other components of the Galaxy is a fundamental step to unveiling the process of Galaxy formation and evolution (Dutra et al. 2002; Wyse et al. 2000).

Despite its importance, the Galactic Bulge GC system remained mostly unexplored, because of the huge foreground extinction, which severely prevents the usage of optical observations. Also, the limited photometric and spectroscopic performances of the previous generations of infrared (IR) arrays, prevented any deep, high resolution imaging and spectroscopy to properly survey the Bulge field and GC population.

Over the last decade a few optical and near IR ground-based photometric studies of bulge GCs have been performed (Ortolani et al. 1996; Guarnieri et al. 1998; Frogel et al. 1995; Davidge 2000). Recently, Valenti et al. (2007) presented homogeneous and accurate IR color-magnitude diagrams (CMDs) of the evolved sequences of a sample of $24 \mathrm{GCs}$ in the Bulge direction.

However, only a few attempts exist so far to estimate the age of Bulge GCs from the direct measurement of the Main Sequence (MS) TO, by using the WFPC2 and NICMOS onboard HST (Ortolani et al. 1995; Heasley et al. 2000; Ortolani et al. 2001; Zoccali et al. 2001; Cohn et al. 2002; Feltzing \& Johnson 2002).

The recent advent of adaptive optics (AO) capabilities on 8m-class telescopes allows unprecedent deep ground-based IR photometry down to the TO level in clusters as distant as $\approx 10 \mathrm{kpc}$ with potential better spatial sampling and coverage than NICMOS onboard HST. We performed a pilot project aimed at measuring the age of the Bulge GCs, by taking advantage of the ESO-VLT NAOS-CONICA (NACO) imaging facility with AO and wavefront sensing in the IR, which is crucial to find natural guide stars bright enough in such a reddened environment. In this Letter, we present the first results of such a project, namely the age determination of the GC NGC 6440. This cluster has an iron abundance $[\mathrm{Fe} / \mathrm{H}]=-0.56$ (Origlia, Valenti \& Rich 2008) and a global metallicity $[\mathrm{M} / \mathrm{H}]=-0.4$ (Valenti et al. 2007) and 
it is located in the inner Bulge at $(1, b)=(7.7,3.8)$ and $8.2 \mathrm{kpc}$ from the Sun. The high reddening $(\mathrm{E}(\mathrm{B}-\mathrm{V})=1.15$, Valenti et al. 2007) along the line of sight yields an extinction of $\approx 3.5$ mag in the visible, and makes this cluster an ideal target to be investigated in the near IR.

\section{Observations and Data Reduction}

The observations have been performed in service mode between August 2005 and September 2006 as part of the programmes 075.D-0359(A) and 077.D-0316(A) (PI: Origlia), by using NACO mounted at the ESO-VLT. We used the S54 camera which offers a relatively large $55^{\prime \prime} \times 55^{\prime \prime}$ FoV with sufficient $\left(0{ }^{\prime \prime} 054 \mathrm{pix}^{-1}\right)$ spatial resolution to resolve the stars. We selected an appropriate field located at $\approx 110 "$ South-West from the cluster center, where crowding is not too severe but the star density is still large enough to sample a reasonable number of stars along the evolved sequences of the CMD (like the Sub Giant Branch). A series of 24 exposures each one 90 sec-long (DIT $=18 \mathrm{sec}$ and NDIT $=5$ and DIT $=10 \mathrm{sec}$ and NDIT=9, in the $\mathrm{J}$ and $\mathrm{H}$ bands, respectively) have been secured. Fig. 1 shows the NACO J and H mosaiced images, with marked the natural guide star used to perform the AO correction.

We used the final mosaiced images obtained from the reduction pipeline and computed PSF-fitting photometry by using STARFINDER (Diolaiti et al. 2000) specifically developed and optimized to obtain accurate photometry in AO-corrected crowded fields. Indeed, STARFINDER allows the modeling of the PSF as a function of the distance from the reference star with a combination of Gaussian/Moffat functions to simultaneously account for the stellar peak and the diffuse seeing halo. More specifically, the complex NACO-PSF in the NGC 6440 images has been modeled with a combination of three Gaussians. Two Gaussians have been used to properly model the central peak of the PSF (their parameters strongly depending on the efficiency of the AO correction), while the third Gaussian parametrizes the PSF halo, and depends on the seeing. Since the AO corrections degrade with the distance from the reference star, STARFINDER has been optimized to allow the parameters of the two Gaussians modeling the central peak to vary as a function of the distance from the reference star. All the parameters of the Gaussians have been fine-tuned by properly modeling a number of high signal-to-noise ratio, unsaturated stars over the entire science image. Once the PSF has been determined, an automatic search of the stars has been performed and the PSF fitting procedure executed for all the detected objects. At the end of this procedure, a final list of instrumental magnitudes and coordinates for more than 2000 stars has been obtained.

Complementary to the NACO observations of the TO region, we have also acquired shallower ( $\approx 1$ min exposures) J,H,Ks images over a wider $\left(5^{\prime} \times 5^{\prime}\right)$ field of view at the ESO- 
NTT using SOFI (programme 081.D-0371(A), PI Valenti) to sample the brightest portion of the CMD, namely the Red Giant Branch (RGB) and the Helium clump. The SOFI J and H images have been reduced by using ROMAFOT (Buonanno et al. 1983) and the instrumental magnitudes have been calibrated and placed on the absolute astrometric system of 2MASS. The brightest, unsaturated stars in the NACO field which are in common with the SOFI dataset have thus been used to transform the NACO J and $\mathrm{H}$ instrumental magnitudes to the SOFI/2MASS photometric system. The average photometric uncertainty turns out to be a few hundreths mag along the $\mathrm{RGB}$ and $\approx 0.1 \mathrm{mag}$ in the TO region. From a direct comparison of our SOFI photometry of the cluster with a control field from 2MASS we find that field contamination along the RGB is below $20 \%$ in the NACO field of view. By performing a number of simulations with the Robin et al. (2003) model of stellar population synthesis of the Galaxy, we also estimate a similar degree of field contamination in the TO region. Hence we conclude that such a degree of field contamination should not appreciably affect our estimates of the Horizontal Branch (HB) and TO levels.

As a template of an old, metal rich GC we took 47 Tuc $([\mathrm{Fe} / \mathrm{H}]=-0.67)$ (Carretta et al. 2000), for which an accurate absolute age estimate (11.2 $\pm 1.1 \mathrm{Gyr})$ has been recently ob-

tained by Gratton et al. (2003). As was done in the case of NGC 6440, we use SOFI shallow photometry of the cluster center to sample the RGB (Origlia et al. 2007) and deep photometry of the TO region as obtained from archival SOFI J,H images of a field located at $\approx 2$ ' from the cluster center (see also Salaris et al. 2007). The photometric analysis of this dataset was performed by using ROMAFOT (Buonanno et al. 1983). The sample has been astrometrized and photometrically calibrated by using the stars in common with the 2MASS catalog.

\section{Results}

Fig. 2 shows the NACO-SOFI $J, J-H$ and $H, J-H$ combined CMDs for NGC 6440. Only stars with $J<16.5$ in the SOFI sample and within 30" from the reference star in the NACO sample are plotted. All the evolutionary sequences from the RGB tip down to the MS are sampled. In particular, the CMDs reach two magnitudes below the cluster TO and offer one of the cleanest insights of the MS TO region ever published for a cluster in the inner Galactic Bulge. These observations show that an AO capability at an $8 \mathrm{~m}$ class telescope is crucial to obtain samples of stars $i) \approx 1$ mag fainter and $i i$ ) poorly affected by incompleteness due to crowding with respect to those obtained from average seeing limited observations. These samples allow us to properly measure the TO region even in severely obscured star clusters in the Galactic Bulge. 
The mean ridge line of the RGB/SGB/MS sequence has been determined by applying an iterative $3 \sigma$-clipping procedure to the observed points and it is overplotted on the CMDs.

The obtained CMDs can be used to measure the age of NGC 6440. As has been well known for many years (Iben 1974; Sandage 1986) the difference in magnitude between the $\mathrm{HB}$ and the TO is a sensitive function of the cluster age for any stellar population older than 2 Gyr. In fact in this age range the luminosity of the HB remains nearly constant while the MS-TO becomes fainter and fainter with increasing age. The $\Delta_{H B-M S T O}$ parameter, defined in different photometric bands, has been successfully used in the past to derive relative ages of Halo (Buonanno et al. 1989; Rosenberg et al. 1999; Chabover et al. 1996) and Bulge (Ortolani et al. 2001) GCs.

In the following we compute this parameter for NGC 6440 and the reference cluster 47 Tuc, in order to derive the relative ages of these two stellar systems.

The HB levels in NGC 6440 turn out to be $\mathrm{J}(\mathrm{HB})=14.70$ and $\mathrm{H}(\mathrm{HB})=13.85$, respectively; for comparison, 47 Tuc shows $\mathrm{J}(\mathrm{HB})=12.5$ and $\mathrm{H}(\mathrm{HB})=12.05$. They have been computed by determining the peak of the HB Luminosity Function for stars with $J<15$ and $(J-$ $H)<0.95$. Such a selection allows a safe determination within \pm 0.05 mag of the mean level of the HB red clump by reducing any possible contamination of RGB stars. The MS TO points, defined as the bluest point along the mean ridge line of the RGB,SGB,MS are located at $\mathrm{J}(\mathrm{TO})=18.8$ and $\mathrm{H}(\mathrm{TO})=18.1$; for comparison, 47 Tuc shows $\mathrm{J}(\mathrm{TO})=16.55$ and $\mathrm{H}(\mathrm{TO})=16.20$. In order to estimate the errors in the TO level determination we follow Meissner \& Weiss (2006). We fitted the ridge line with a fifth-order $(\mathrm{J}-\mathrm{H})=\mathrm{f}(\mathrm{J})$ polynomial and compute the $\sigma\left(\mathrm{J}_{\mathrm{TO}}\right)=\sigma(\mathrm{f}(\mathrm{J})) / \sqrt{(\mathrm{N})}$, where $\sigma(\mathrm{f}(\mathrm{J}))$ is the standard deviation of $\mathrm{f}(\mathrm{J})$ within a $3 \sigma(\mathrm{J}-\mathrm{H})_{\text {TO }}$ color interval around the TO. The inferred $\sigma\left(\mathrm{J}_{\mathrm{TO}}\right)$ are 0.07 for NGC 6440 and 0.06 for 47 Tuc. As an independent estimate we also compute the distribution of the $\mathrm{J}_{\mathrm{TO}}$ values obtained by directly fitting the observed CMD TO region with a fifth-order polynomial, by varying the magnitude interval around the TO and the $\sigma$ rejection threshold between 2 and 4. The obtained distributions of the TO level determinations are plotted in Fig. 3. The best fit Gaussian $\sigma$ turns out to be 0.08 in NGC 6440 and 0.05 in 47 Tuc. We thus conservatively assume a $\pm 0.1 \mathrm{mag}$ error in NGC 6440 and $\pm 0.05 \mathrm{mag}$ in $47 \mathrm{Tuc}$. On the basis of these measurements, the magnitude difference between the HB and the TO in the $\mathrm{J}$ band of NGC 6440 is $\Delta \mathrm{J}(\mathrm{HB}-\mathrm{TO})=4.10 \pm 0.11$ and the corresponding value in 47 Tuc is $\Delta \mathrm{J}(\mathrm{HB}-\mathrm{TO})=4.05 \pm 0.07$. These values suggest a similar age for the two clusters. The nice similarity between the CMDs of the two clusters is clearly visible in Fig. 4, where the CMD of 47 Tuc (right panel) has been shifted in color and magnitude according to the reddening and distance of NGC 6440, and to match the HB level of the latter (left panel). Note that the metallicity of NGC 6440 is slightly higher $(\approx 0.1 \mathrm{dex})$ than that of 47 Tuc. 
However, inspection of the theoretical isochrones suggests that a $\delta[\mathrm{Fe} / \mathrm{H}] \approx 0.1$ translates into a $\delta \mathrm{J}(\mathrm{HB}-\mathrm{TO}) \approx 0.03$, only, which is well within the errors. Hence, from this analysis we conclude that NGC 6440 is consistent with being coeval to 47 Tuc, that is $\approx 11$ Gyr old.

In order to evaluate the overall uncertainty of the $\triangle J(H B-T O)$ method in deriving absolute ages, we use theoretical isochrones of different ages. Two sets of $\alpha$-enhanced models at $[\mathrm{M} / \mathrm{H}]=-0.65$ and $[\mathrm{M} / \mathrm{H}]=-0.35$, respectively, to encompass the metallicity of 47 Tuc and NGC 6440, and ages ranging from 2 to 13 Gyr, have been retrieved from the BASTI database (Pietrinferni et al. 2004) and transformed into the observational 2MASS (J,J-H) plane following the prescriptions by Origlia \& Leitherer (2000). The difference between the mean value of the HB and the TO level was computed for each isochrone 1 . We find that in the metallicity range under consideration, $\mathrm{a} \approx 0.1 \mathrm{mag}$ error in the $\Delta \mathrm{J}(\mathrm{HB}-\mathrm{TO})$ value translates into a 2 Gyr uncertainty in the 8-11 Gyr age range and $\geq 3$ Gyr between 10 and 13 Gyr, since $\Delta \mathrm{J}(\mathrm{HB}-\mathrm{TO})$ becomes progressively less and less sensitive to age above 11 Gyr. Hence we can conclude that NGC-6440 is $11_{-2}^{+3}$ Gyr, similar to 47 Tuc and other metal rich GCs of the Galaxy.

\section{Discussion and Conclusions}

In this letter we have presented the age determination for NGC 6440: it is the first time that ground based AO imaging in the near infrared has been used to successfully measure the TO region and derive the age of a Bulge GC. The few previous studies have been all based on space observations. In fact, since the pioneering work by Ortolani et al. (1995) who first measured the TO region of NGC 6553 and NGC 6528 by using HST-WFC2 and suggested a near-coeval age for the Galactic bulge and Halo, a number of subsequent works using HST and the WFPC2 and NICMOS imagers (Ortolani et al. 2001; Feltzing \& Johnson 2002) refined techniques and procedures to measure the age of these two globular clusters, by also applying proper motion decontamination to the CMDs (Zoccali et al. 2001; Feltzing \& Johnson 2002). Only one other cluster in the inner Bulge, namely Terzan 5 (Ortolani et al. 2001; Cohn et al. 2002) has been studied with HST-NICMOS, while Heasley et al. (2000) analyzed two other GCs observed with HST-WFPC2 in the outer Bulge, namely NGC 6624 an NGC 6637. All

\footnotetext{
1 As an independent test of the isochrones, we can also use our measurement, we can also use our measurement of the $\Delta \mathrm{J}(\mathrm{HB}-\mathrm{TO})$ in 47 Tuc and its robust age estimate by Gratton et al. (2003) to evaluate the reliability of the theoretical $\Delta \mathrm{J}(\mathrm{HB}-\mathrm{TO})$ in deriving absolute ages of a stellar system. By using the $[\mathrm{M} / \mathrm{H}]=-0.65$ isochrone, we find that 47 Tuc turns out to be $\approx 10$ Gyr old, which is $\approx 1$ Gyr younger than in Gratton et al. (2003). In this context, the $\Delta \mathrm{J}(\mathrm{HB}-\mathrm{TO})$ value measured in NGC 6440 would give ages $\approx 11$ and $\approx 10$ Gyr, by using the $[\mathrm{M} / \mathrm{H}]=-0.65$ and the $[\mathrm{M} / \mathrm{H}]=-0.35$ isochrones, respectively.
} 
these studies suggest that these clusters are old (>10 Gyr) and likely coeval with 47 Tuc.

While our and previous studies agree on the fact that Bulge GCs are likely coeval to 47 Tuc, absolute age estimates range between 11 and 14 Gyr, depending on the adopted theoretical isochrones and/or the absolute age for 47 Tuc itself. In this respect, a significant step forward in setting the absolute age of reference clusters has been performed by Gratton et al. (2003) who provided accurate distances and absolute ages for three pillar GCs, namely NGC 6397, NGC 6752 and 47 Tuc, by using the MS Fitting Method and the Hipparcos distance scale. Their age estimate for 47 Tuc turns out to be $11.2 \pm 1 \mathrm{Gyr}, \approx 2.6 \mathrm{Gyr}$ younger than the other more metal poor Halo clusters. A somewhat younger age (by 1-2 Gyr) was previously suggested by Rosenberg et al. (1999), who also found slightly younger ages for other two metal rich clusters in their sample, namely NGC 6352 and NGC 6838, which possibly belong to the thick disk population, like 47 Tuc (see also Figure 2 in Ferraro 2004).

These first set of age determinations seem to suggest a common epoch of formation of the tick disk and Bulge and an overall GC formation process in our Galaxy starting $\approx 13.5$ Gyr ago (Gratton et al. 2003) with the formation of the oldest Halo GCs and ending $\approx 11$ Gyr ago with the formation of the metal rich objects in the thick disk and Bulge. In this scenario, the few younger GCs found in the Halo (like for example Pal 12, Arp 2, Terzan 8 etc.) could not have formed in situ. In fact, a number of recursive accretions of satellites and their GC systems (as e.g. the Sagittarius Dwarf Spheroidal, Ibata,Gilmore \& Irwin 1994) could have significantly contributed to form the present Halo stellar populations (Bellazzini, Ferraro \& Ibata 2003; Ferraro 2004).

However, a larger sample of both Bulge and thick disk GCs urgently need to be observed with the purpose of determining their relative and absolute ages, before drawing firm conclusions. This is something that is well within the capabilities of the current generation of instruments, such as the refurbished HST and the improving performances of ground based AO imagers.

This research was supported by the Ministero dell'Istruzione, dell'Universitá e della Ricerca and by the Progetti Strategici 2006 granted by the University of Bologna. We thank the anonymous Referee for his/her helpful comments.

\section{REFERENCES}

Ballero, S. K., Matteucci, F., Origlia, L., \& Rich, R. M. 2007, A\&A, 467, 123 
Bellazzini, M., Ferraro, F. R., \& Ibata, R. 2003, AJ, 125, 188

Buonanno, R., Buscema, G., Corsi, C. E., Ferraro, I., \& Iannicola, G. 1983, A\&A, 126, 278

Buonanno, R., Corsi, C. E., \& Fusi Pecci, F. 1989, A\&A, 216, 80

Carretta, E. Gratton, R. G., Clementini, G., \& Fusi Pecci, F. 2000, ApJ, 533, 215

Chaboyer, B., Demarque, P., Kernan, P. J., Krauss, L. M., \& Sarajedini, A. 1996, MNRAS, 283,683

Cohn, H. N., Lugger, P. M., Grindlay, J. E., \& Edmonds, P. D. 2002, ApJ, 571, 818

Davidge, T. J. 2000, ApJS, 126, 105

De Angeli, F., Piotto, G., Cassisi, S., Busso, G., Recio-Blanco, A., Salaris, M., Aparicio, A., \& Rosenberg, A. 2005, AJ, 130, 116

Diolaiti, E., Bendinelli, O., Bonaccini, D., Close, L., Currie, D., \& Parmeggiani, G. 2000, A\&AS, 147, 335

Dutra, C. M., Santiago, B. X., \& Bica, E. 2002, A\&A, 381, 219

Feltzing, s., \& Johnson, R. A. 2002, A\&A, 385, 67

Ferraro, F. R. 2004, inHow does the Galaxy Work?, E.J. Alfaro, E. Perez and J. Franco eds., Kluwer Academic Publishers, 241

Ferraro, F. R., Valenti, E., \& Origlia, L. 2006, ApJ, 649, 243

Frogel, J. A., Kuchinski, L. E., \& Tiede, G. P. 1995, AJ, 109, 1154

Gratton, R. G., Bragaglia, A., Carretta, E., Clementini, G., Desidera, S., Grundahl, F., \& Lucatello, S. 2003, A\&A, 408, 529

Guarnieri, M. D., Ortolani, S., Montegriffo, P., Renzini, A., Barbuy, B., Bica, E., \& Moneti, A. 1998, A\&A, 331, 70

Ibata, R. A., Gilmore, G., \& Irwin, M. J. 1994, Nature, 370, 194

Heasley, J. N., Janes, K. A., Zinn, R., Demarque, P., Da Costa, G. S., \& Christian, C. A. 2000, AJ, 120, 879

Iben, I. 1974, Late Stages of Stellar Evolution, 66, 237 
Matteucci, F., Romano, D., \& Molaro, P. 1999, A\&A, 341, 458

McWilliam, A. 1997, ARA\&A, 35, 503

Meissner, F., \& Weiss, A. 2006, A\&A, 456, 1085

Origlia, L., \& Leitherer, C. 2000, AJ, 119, 2018

Origlia, L., Rood, R. T., Fabbri, S., Ferraro, F. R., Fusi Pecci, F., \& Rich, R. M. 2007, ApJ, 667,85

Origlia, L., Valenti, E., \& Rich, R. M. 2008, MNRAS, in press (astro-ph/0805-3442)

Ortolani, S., Renzini, A., Gilmozzi, R., Marconi, G., Barbuy, B., Bica, E., \& Rich, R. M. 1995, Nature, 377, 701

Ortolani, S., Barbuy, B., \& Bica, E. 1996, A\&A, 308, 733

Ortolani, S., Barbuy, B., Bica, E., Renzini, A., Zoccali, M., Rich, R. M., \& Cassisi, S. 2001, A\&A, 376, 878

Pietrinferni, A, Cassisi, S., Salaris, M., \& Castelli F. 2004, AJ, 612, 168

Robin, A. C., Reyle, C., Derriere, S., \& Picaud, S. 2003, A\&A, 409, 523

Rosenberg, A., Saviane, I., Piotto, G., \& Aparicio, A. 1999, AJ, 118, 2306

Rosenberg, A. 2000, PASP, 112, 575

Salaris, \& Weiss, A. 2002, A\&A, 388, 492

Salaris, M., Held, E. V., Ortolani, S., Gullieuszik, M., \& Momany, Y. 2007, A\&A, 476, 243

Sandage, A. 1986, ARA\&A, 24, 421

Valenti, E., Ferraro, F. R., \& Origlia, L. 2007, AJ, 133, 1287

Wyse, R. F. G. 1999, Ap\&SS, 267, 145

Wyse, R. F. G., Gilmore, G., Norris, J. E., \& Freeman, K. C. 2000, Bulletin of the American Astronomical Society, 197, 424

Zoccali, M., Renzini, A., Ortolani, S., Bica, E., \& Barbuy, B. 2001, AJ, 121, 2638 

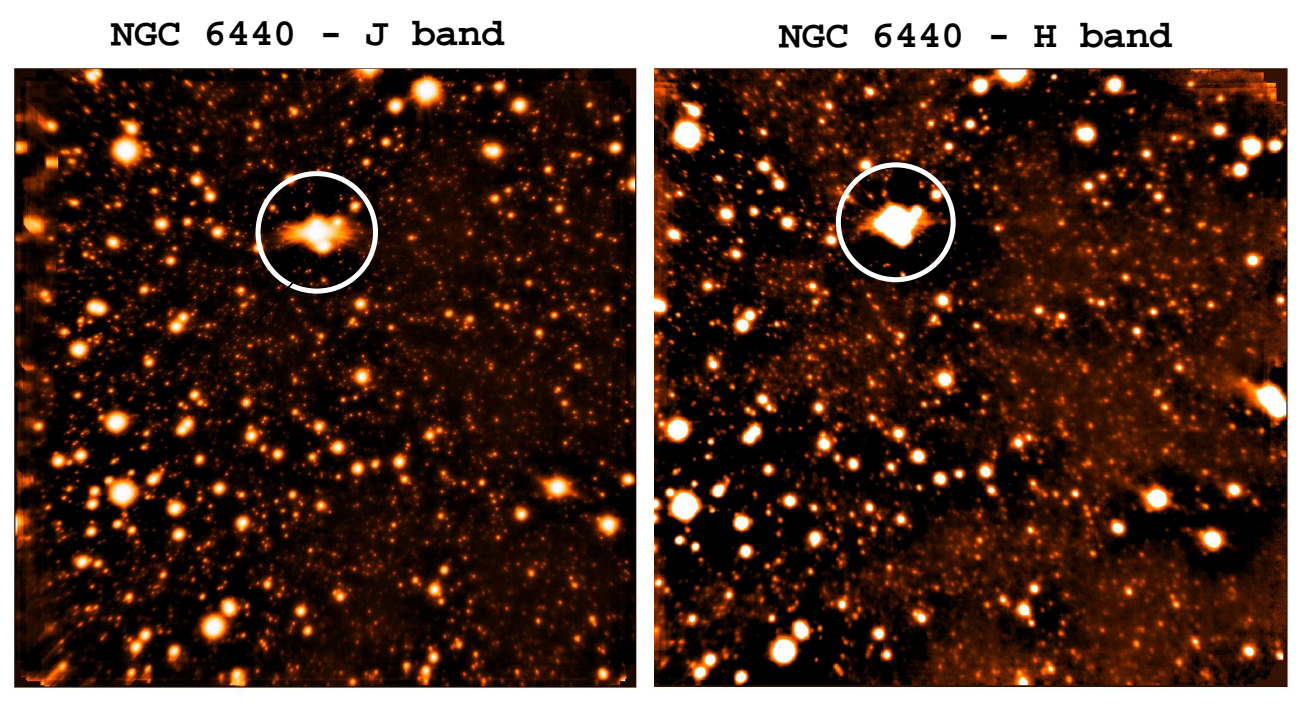

Fig. 1. - NACO J and H band images of the observed field $(\approx 50 " \times 50 ")$ located $\approx 110 "$ South-West from the NGC 6440 center. North is up, East is left. The circled star is the natural guide star used for $\mathrm{AO}$ correction. 

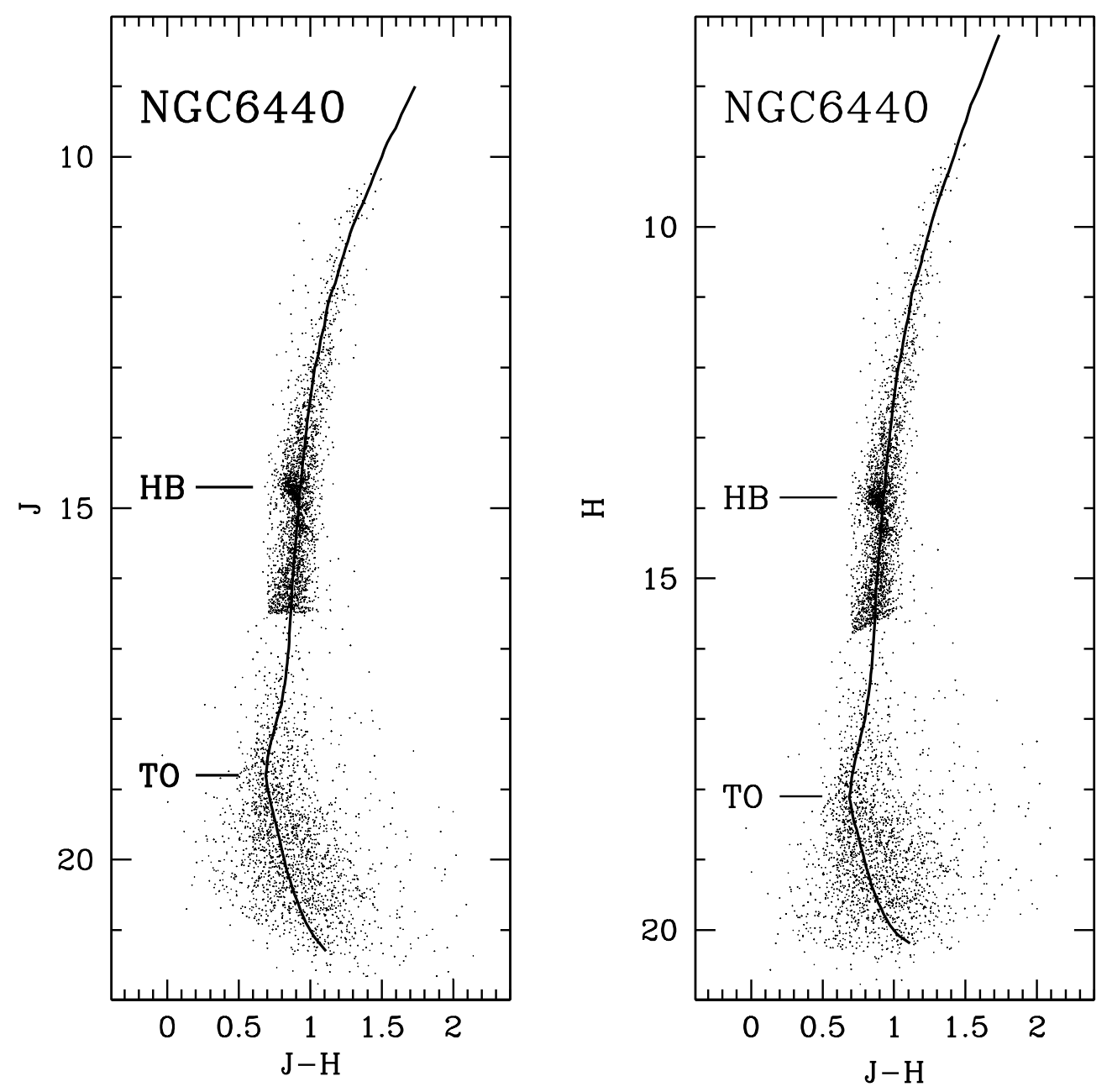

Fig. 2.- Combined NACO-SOFI (J,J-H) and (H,J-H) CMDs for NGC 6440. Only stars with $J<16.5$ from the SOFI sample and with $r<30$ " from the reference star in the NACO sample are plotted. The RGB-SGB-MS mean ridge lines are overplotted to the data. The $\mathrm{HB}$ and TO levels are also marked for reference. 


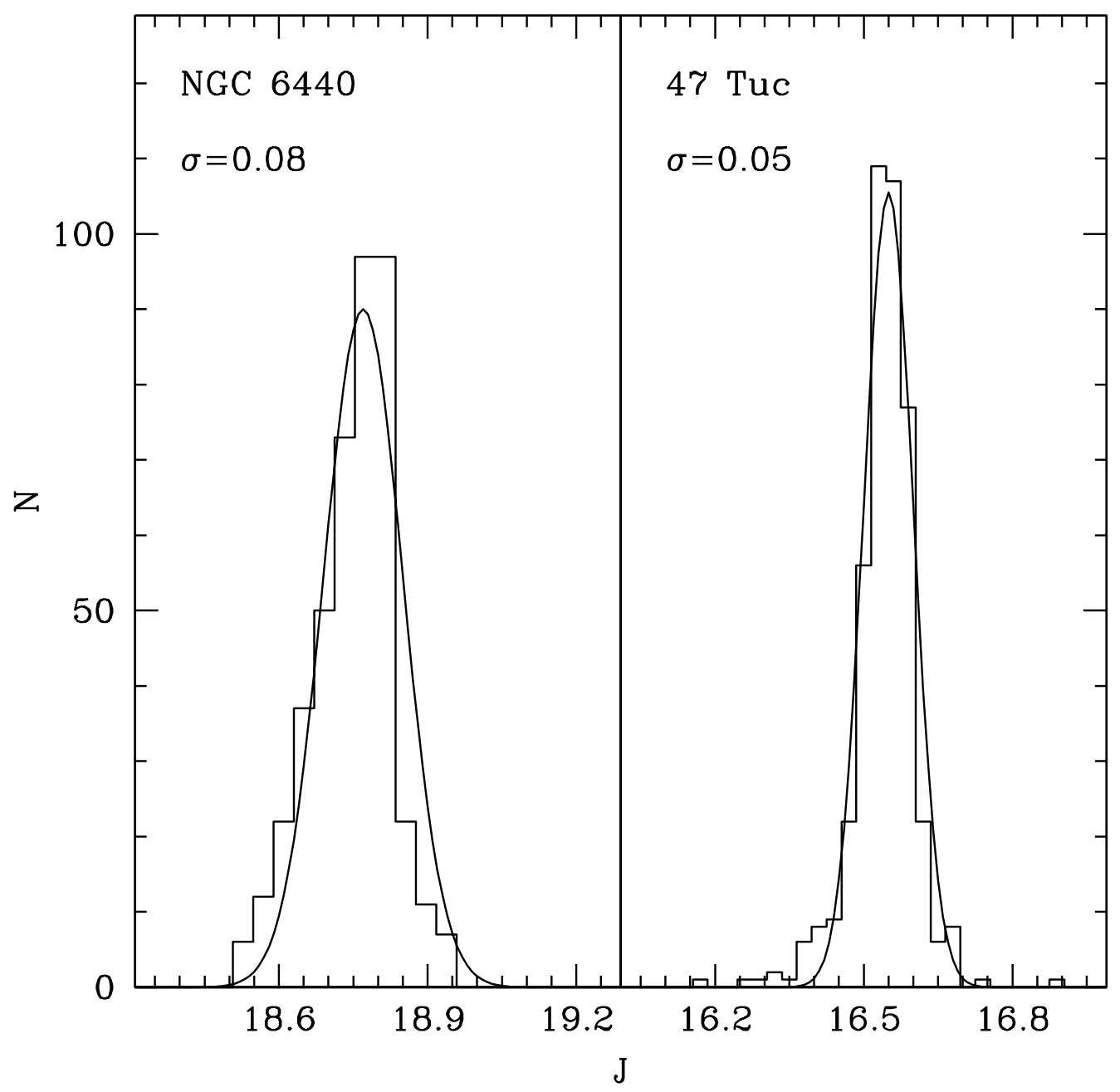

Fig. 3. - Distribution of the TO level determinations as obtained from $\approx 400$ fitting models of the TO region for NGC 6440 (left panel) and 47 Tuc (right panel). The Gaussian best fit and the corresponding $\sigma$ value for each distribution is also reported. 


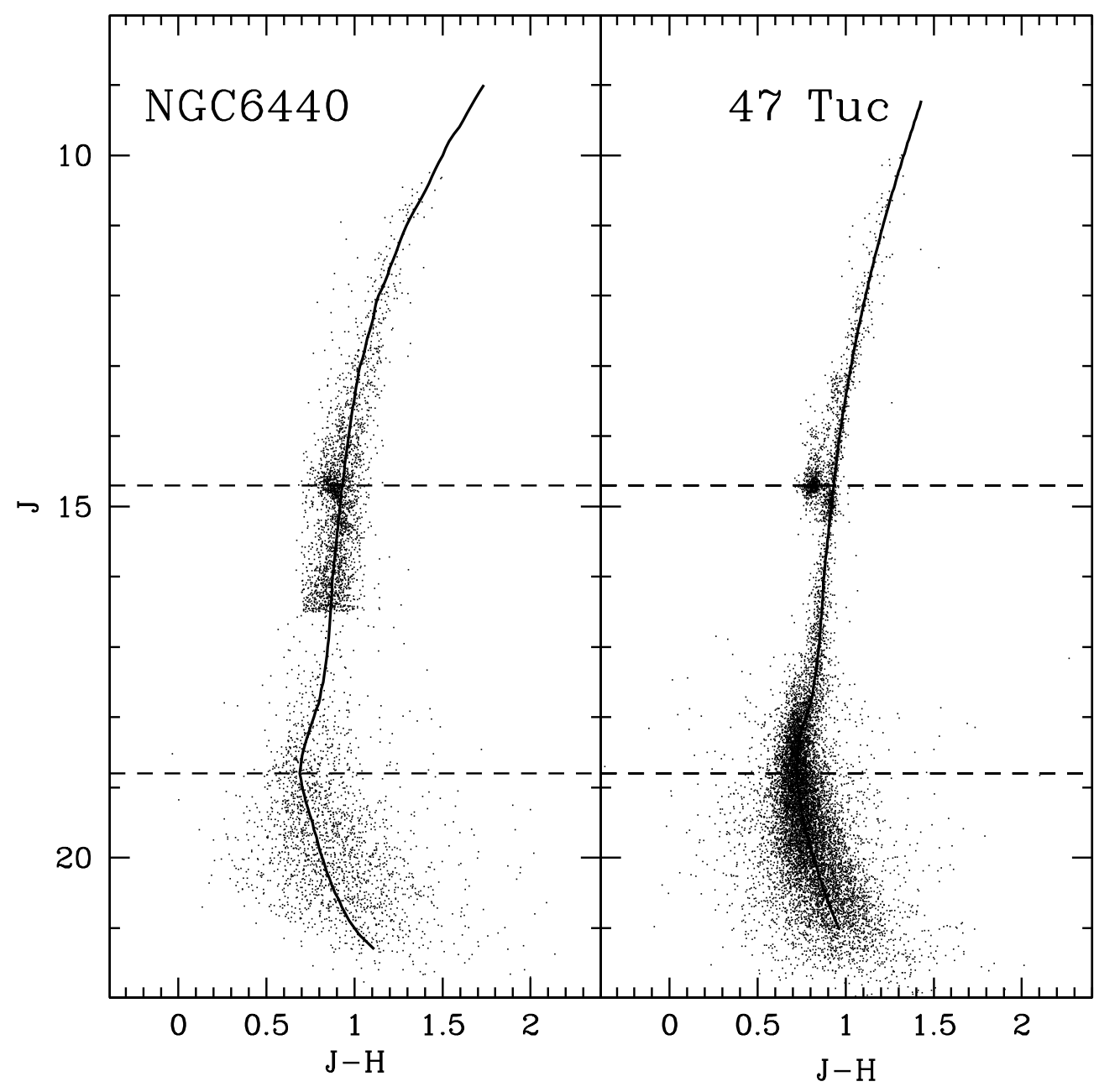

Fig. 4.- The $(J, J-H)$ CMDs for NGC 6440 (left panel) and 47 Tuc (right panel), as shifted in color and magnitude, accordingly to the reddening and distance modulus of NGC 6440. The horizontal dashed lines mark the HB and TO level of NGC 6440. 